\title{
Rosuvastatin pharmacogenetics in African populations
}

\author{
Nyarai Desiree Soko1, Collen Masimirembwa² \& Collet Dandara*,3 \\ ${ }^{1}$ Department of Biochemistry, Faculty of Science, University of Zimbabwe, Harare, Zimbabwe \\ ${ }^{2}$ African Institute of Biomedical Science \& Technology (AiBST), DMPK Department, Wilkins Hospital, Corner Rekai Tangwena \& \\ Josiah Tongogara Avenue, Harare, Zimbabwe \\ ${ }^{3}$ Department of Pathology, Division of Human Genetic, Faculty of Health Sciences, University of Cape Town, Cape Town, South \\ Africa \\ *Author for correspondence: Tel.: +27 (0)21 4066506; collet.dandara@uct.ac.za
}

"observations in this short communication make a case for the need to include African populations where possible, of different intra-African ethnicities, in clinical trials for drug development and discovery so that underlying genetic differences that affect drug response are picked up in the early stages of drug development"”

First draft submitted: 18 October 2018; Accepted for publication: 18 October 2018; Published online: 6 November 2018

Keywords: 3-hydroxymethylglutaryl co-enzyme A reductase • ABCG2• African populations • cholesterol • LDL • rosuvastatin $\bullet$ SLCO1B1

Rosuvastatin is an inhibitor of 3-hydroxymethylglutaryl co-enzyme A reductase. Inhibition of 3hydroxymethylglutaryl co-enzyme A reductase by rosuvastatin leads to reduced production of mevalonic acid by hepatocytes. Consequently, the overall effect is reduction of total circulating levels of cholesterol and low-density lipoprotein in the blood. Rosuvastatin, is thus indicated in the treatment of hypercholesterolemia, hypertriglyceridemia, hyperlipidemia, mixed dyslipidemia and homozygous familial hypercholesterolemia [1].

Pharmacokinetic (PK) studies of rosuvastatin show that both PK profiles and PK parameters such as area under the curve and maximum drug plasma concentration (Cmax) are similar in Caucasian [2,3] and African individuals [4]. However, a shorter time to reach Cmax, indicative of faster rates of hepatocyte absorption of rosuvastatin is observed in individuals of African descent [4]. PK parameters of rosuvastatin in individuals of Asian descent; however, are persistently different from both Caucasian and African individuals. Both area under the curve and Cmax are consistently twice as high in participants of Asian descent $[2,3]$ than they are in Caucasian and African individuals. Consequently, these interethnic differences have led to a reduction in starting dose for patients of Asian descent [1]. Based on these interethnic variations, it becomes important to evaluate if the observed PK variability is caused by the same factors in the different ethnic populations and especially tease out the contribution of pharmacogenetic factors.

Traditionally, the pharmacogenetics of rosuvastatin has been dominated by variation in two genes, SLCO1B1 and $A B C G 2$ and specifically two SNPs, namely, SLCO1B1 c.521T>C (rs4149056, p.Val174Ala) and $A B C G 2$ c.421 (rs2231142, p.Gln141Lys). SLCO1B1 encodes the polypeptide OATP1B1, an influx transporter expressed in the liver and intestines. OATP1B1 is responsible for the uptake of rosuvastatin from the intestinal lumen by enterocytes and uptake of rosuvastatin by hepatocytes. Therefore, polymorphisms that alter OATP1B1 activity affect rosuvastatin plasma levels. Indeed, the SNP SLCO1B1 c.521T >C has been shown to functionally reduce OATP1B1 transporter activity [5] and hence increase plasma rosuvastatin concentrations/exposure leading to increased risk of statin-induced myopathy [6]. ABCG2 on the other hand, encodes the efflux transporter BCRP which is constitutively expressed on the canicular membrane of hepatocytes and in enterocytes. BCRP actively pumps rosuvastatin back into enterocytes from portal blood resulting in fecal excretion of the drug. BCRP pumps rosuvastatin out of the liver for biliary excretion. Similarly, alterations to BCRP activity have a bearing on the amount of rosuvastatin circulating in the blood. The polymorphism $A B C G 2$ c. $421 \mathrm{C}>\mathrm{A}$ is consistently associated with increased risk of simvastatin induced myopathy (SIM) [7] due to increased plasma concentrations of rosuvastatin [8].

Future $\because \%$ Medicine 
Table 1. African specific variants implicated in rosuvastatin pharmacokinetics variability

\begin{tabular}{|c|c|c|c|c|c|c|c|c|}
\hline \multirow{2}{*}{$\begin{array}{l}\text { Associated } \\
\text { phenotype }\end{array}$} & \multirow[t]{2}{*}{ Gene } & \multicolumn{3}{|c|}{ Polymorphisms } & \multicolumn{4}{|c|}{ Minor allele frequency } \\
\hline & & dbSNP ID & $\begin{array}{l}\text { Nucleotide } \\
\text { change }\end{array}$ & $\begin{array}{l}\text { Amino acid } \\
\text { change }\end{array}$ & Zimbabwean (9) & African $^{\dagger}$ & Caucasian $^{\dagger}$ & Asian $^{\dagger}$ \\
\hline \multirow{5}{*}{$\begin{array}{l}\text { Increased } \\
\text { rosuvastatin } \\
\text { plasma } \\
\text { concentration }\end{array}$} & SLCO1B1 & rs4149056 & c.521T $>C$ & p.V174A & 0.00 & 0.02 & $0.20(12)$ & $0.15(12)$ \\
\hline & $A B C G 2$ & rs2231142 & c. $421 \mathrm{C}>\mathrm{A}$ & p.N130D & 0.00 & $0.002(9)$ & $0.17(3)$ & $0.38(3)$ \\
\hline & $A B C C 2$ & rs8187692 & c. $3542 \mathrm{G}>\mathrm{A}$ & p.R1181Q & 0.42 & 0.12 & 0.00 & 0.00 \\
\hline & HNF4A & rs3212207 & g.73174G $>C$ & Intronic & 0.17 & 0.03 & 0.00 & 0.00 \\
\hline & FOXA3 & rs16980091 & c. $516 \mathrm{C}>\mathrm{T}$ & $\mathrm{N} 172 \mathrm{~N}$ & 0.58 & 0.31 & 0.11 & 0.09 \\
\hline \multirow{5}{*}{$\begin{array}{l}\text { Decreased } \\
\text { rosuvastatin } \\
\text { plasma } \\
\text { concentration }\end{array}$} & SLCO1B1 & rs11045854 & c. $882 \mathrm{G}>\mathrm{A}$ & L294L & 0.17 & 0.03 & 0.00 & 0.00 \\
\hline & & rs12305884 & g. $64545 \mathrm{G}>\mathrm{A}$ & Intronic & 0.17 & 0.10 & 0.00 & 0.00 \\
\hline & $A B C C 2$ & rs17216198 & c. $1219 C>T$ & L407L & 0.17 & 0.03 & 0.00 & 0.00 \\
\hline & & rs7899457 & c. $3831 \mathrm{C}>\mathrm{T}$ & L1370L & 0.17 & 0.11 & 0.00 & 0.00 \\
\hline & & rs8187706 & c. $3714 \mathrm{G}>\mathrm{A}$ & E1470E & 0.17 & 0.12 & 0.00 & 0.00 \\
\hline
\end{tabular}

${ }^{\dagger}$ Minor allele frequencies obtained from 1000 genomes unless otherwise stated.

Pharmacogenomic studies show varied frequencies of both the minor alleles of SLCO1B1 rs4149056 and ABCG2 rs2231142 (Table 1). SLCO1B1 rs4149056 has a higher frequency in populations of Caucasian descent (0.20) [3] than those of Asian descent (0.15) and the frequency is low and heterogeneous among Africans [9], ranging from 0 to $13 \%$. While $A B C G 2$ rs 2231142 has a higher frequency in Asian populations (0.38) [3] when compared with populations of Caucasian descent (0.17) [3] while rare in African descendant populations (0.002-0.005) [9]. This implies that interethnic variability between Asian and Caucasian individuals could be influenced by the presence of $A B C G 2$ rs2231142. It also implies SLCO1B1 rs 4149056 may play a reduced role in the pharmacogenetics of rosuvastatin in Asian individuals while playing a varied role in individuals of African individuals.

The rarity of both SLCO1B1 rs4149056 and ABCG2 rs2231142 in individuals of African descent point to possible limited variability of rosuvastatin exposure among Africans. However, observed data on the PKs of rosuvastatin in African individuals [4] point to a high interindividual variability that can be explained by neither SLCO1B1 rs4149056 nor $A B C G 2$ rs2231142. Such results indicate a possibility of other pharmacogenes or environmental factors playing significant roles in the PKs of rosuvastatin among Africans or possibility of an African specific mosaic of variants contributing to the observed interindividual variability. Interestingly, within African populations [9], frequencies of $S L C O 1 B 1$ rs 4149056 and $A B C G 2$ rs2231142 differ from one ethnic group to another. Therefore, the traditional blanket approach to interethnic pharmacogenetics where data from one African group can be extrapolated to all individuals of African descent cannot be applied blindly if safety and efficacy of drugs is to remain the goal of pharmacogenomics. "When treating individual patients, the focus must be shifted from populations, ethnicities or races to the inherent genetic individuality that results from unique mosaics of variable haplotypes," this statement made by Suarez-Kurtz [10], is an apt analysis of the pharmacogenetics of rosuvastatin in African populations. Our previous data [9], on African ethnic groups, show that the frequencies of SLCO1B1 rs4149056 variants is low. Thus, when treating individual patients there should be a shift from predominantly ethnic populations to focus on individual variant mosaics if efficacy and safety are to be attained at individual level.

Using data from individuals of wholly African descent, involved in a rosuvastatin PK trial [9]; we identified a different set of African-specific variants (Table 1). These African specific variants were significantly associated with rosuvastatin PKs variability among African populations. These variants do require further characterization in an effort to provide a comprehensive understanding of their effect on the PK of rosuvastatin. Such efforts to further understand the interindividual variation of rosuvastatin PKs should take into account environmental factors such as transporter inducers and inhibitors. The use of statins in treating dyslipidemia induced by HIV or protease inhibitors is on the increase in Africa making this drug of importance to these populations that carry the highest burden of millions of patients on antiretroviral treatment. 
The burden of disease in the African continent is laden with both cardiovascular diseases and communicable diseases such as HIV/AIDS, tuberculosis and malaria. Both conditions contribute significantly to raise plasma cholesterol levels. Rosuvastatin lacks significant CYP450 metabolism [11], therefore, it attracts the attention of clinicians treating patients with HIV-associated dyslipidemia as it lacks drug-to-drug potential. Use of rosuvastatin on the African continent is therefore expected to grow as the burden of cardiovascular diseases increase owing to change in lifestyles across the continent and the burden of HIV-associated dyslipidemia linked to antiretroviral treatment escalates. Data from Africa points to inter-African pharmacogenetic variability coupled by a different set of pharmacogenetic polymorphisms affecting rosuvastatin pharmacology when compared with both Asian and Caucasian populations. These observations call for improved genomic characterization of different African populations to discover the remaining genetic contributors to differential drug response. In addition, the observations in this short communication make a case for the need to include African populations where possible, of different intra-African ethnicities, in clinical trials for drug development and discovery so that underlying genetic differences that affect drug response are picked up in the early stages of drug development.

\section{Financial \& competing interests disclosure}

The authors have no relevant affiliations or financial involvement with any organization or entity with a financial interest in or financial conflict with the subject matter or materials discussed in the manuscript. This includes employment, consultancies, honoraria, stock ownership or options, expert testimony, grants or patents received or pending, or royalties.

No writing assistance was utilized in the production of this manuscript.

\section{References}

1. Astra Astra. Crestor prescribing information (2016). https://www.crestor.com/cholesterol-medicine/how-when-to-take-crestor.html

2. Lee E, Ryan S, Birmingham B et al. Rosuvastatin pharmacokinetics and pharmacogenetics in white and Asian subjects residing in the same environment. Clin. Pharmacol. Ther. 78(4), 330-41 (2005).

3. Birmingham BK, Bujac SR, Elsby R et al. Impact of ABCG2 and SLCO1B1 polymorphisms on pharmacokinetics of rosuvastatin, atorvastatin and simvastatin acid in Caucasian and Asian subjects: a class effect? Eur. J. Clin. Pharmacol. 71(3), 341-55 (2015).

4. Soko N, Dandara C, Ramesar R, Kadzirange G, Masimirembwa C. Pharmacokinetics of rosuvastatin in 30 healthy Zimbabwean individuals of African ancestry. Br. J. Clin. Pharmacol. 82(1), 326-328 (2016).

5. Tirona RG, Leake BF, Merino G, Kim RB. Polymorphisms in OATP-C: identification of multiple allelic variants associated with altered transport activity among European- and African-Americans. J. Biol. Chem. 276(38), 35669-75 (2001).

6. Pasanen MK, Neuvonen M, Neuvonen PJ, Niemi M. SLCO1B1 polymorphism markedly affects the pharmacokinetics of simvastatin acid. Pharmacogenet. Genomics 16(12), 873-9 (2006).

7. DeGorter MK, Tirona RG, Schwarz UI et al. Clinical and pharmacogenetic predictors of circulating atorvastatin and rosuvastatin concentrations in routine clinical care. Circ. Cardiovasc. Genet. 6(4), 400-8 (2013).

8. Keskitalo JE, Zolk O, Fromm MF, Kurkinen KJ, Neuvonen PJ, Niemi M. ABCG2 polymorphism markedly affects the pharmacokinetics of atorvastatin and rosuvastatin. Clin. Pharmacol. Ther. 86(2), 197-203 (2009).

9. Soko ND, Chimusa E, Masimirembwa C, Dandara C. An African-specific profile of pharmacogene variants for rosuvastatin plasma variability: limited role for SLCO1B1 c.521T>C and ABCG2 c.421A >C. Pharmacogenomics J. (2018).

10. Suarez-kurtz G. Ethnic differences in drug therapy: a pharmacogenomics perspective. Expert Rev Clin Pharmacol. 1(3), 337-339 (2008).

11. Cooper KJ, Martin PD, Dane AL, Warwick MJ, Raza A, Schneck DW. The effect of erythromycin on the pharmacokinetics of rosuvastatin. Eur. J. Clin. Pharmacol. 59, 51-6 (2003).

12. Niemi M, Pasanen MK, Neuvonen PJ. Organic anion transporting polypeptide 1B1: a genetically polymorphic transporter of major importance for hepatic drug uptake. Pharmacol. Rev. 63(1), 157-81 (2011). 
